Una negociación sin precedentes: ¿un santo legado impopular? La diplomacia presidencial como elemento legitimador del acuerdo con las Farc.

\author{
Carolina Serna Jaramillo* Isabella Marín Jiménez ${ }^{\star *}$ \\ ** Abogada. (e) Especialización en Derecho Laboral y de la Seguridad Social. \\ Docente investigadora de tiempo completo Universidad de San Buenaventura \\ Cali. \\ **Estudiante de Gobierno y RRII Representante de la Facultad de derecho y \\ ciencias políticas de la USB.
}

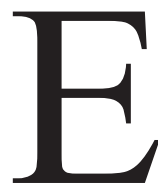
1 ex ministro de defensa, Juan Manuel Santos Calderón, asumió la presidencia de la República de Colombia en el año 2010, atendiendo al continuismo de la política de seguridad democrática del expresidente Álvaro Uribe Vélez; sin embargo, al sumar todos los esfuerzos en pro de la consolidación de la paz y al alejarse de una agenda totalmente securitizada, terminó su gobierno envuelto en una serie de opiniones polarizadas y un aparente índice de popularidad alrededor de 37\%, según las encuestas aplicadas por Yanhaas, Gallup Pull e Ipsos Public Afairs en 2018, debido en gran parte a la ruptura entre la Unidad Nacional y el Centro Democrático, el cual se constituyó como cabeza de una oposición acérrima a su gobierno.

Aproximadamente desde los años 30, Colombia ha padecido una guerra bipartidista conocida como el Conflicto Armado Interno Colombiano, en adelante (CAI) y una vez conformados los grupos al margen de la ley se conoce uno de ellos como la guerrilla de las Farc (Fuerzas Armadas Revolucionarias de Colombia); sin embargo, el proceso de negociación que abriría las puertas a una Colombia en paz, desconoció la existencia de otros actores fundamentales para la terminación del conflicto más antiguo de América Latina. Para muestra de lo anterior, es inexplicable el haber llamado "Proceso de Paz" a la negociación con este grupo ilegal dejando de lado otros como el Ejército de Liberación Nacional - ELN.

Desde el primer mandato del ex presidente López Pumarejo (1934-1938), hasta el de Andrés Pastrana Arango (1998-2002) no hubo un sólo presidente que propusiera una iniciativa semejante a un proceso de negociación llevado a cabo a pesar de las muchas acciones atroces y los diferentes sucesos infortunados que generaron que el grupo de las Farc comenzara a ser denominado "grupo terrorista" por actores de relevancia 
internacional como la Unión Europea y los Estados Unidos.

A pesar de ello, Pastrana decidió no clasificar a las Farc como tal y más bien reconocerlo como un actor político con el cual iniciar las primeras negociaciones legítimas en el Caguán (1998-2001) y que a su vez, estas fueran apoyadas en la esfera nacional e internacional. Las estrategias de internacionalización de este proceso se orientaron a encontrar apoyos económicos para las regiones devastadas por la violencia; no obstante, los 500 millones de dólares concedidos por Estados Unidos, en el marco del Plan Colombia (1999), estuvieron direccionados en mayor medida a la lucha contra el problema de las drogas y al fortalecimiento de las Fuerzas Armadas del país, que al mismo proceso de negociación. Es imposible olvidar la imagen del presidente -ingenuo- sentado, sin aliados, en una mesa de negociación con un actor que se debilitaba ideológicamente, pero que presentaba un escalonamiento sistemático cimentado en la delincuencia organizada y el narcotráfico.

Con el ascenso al poder de Álvaro Uribe Vélez en 2002 y el establecimiento de una política altamente securitizada y de "mano dura" contra las amenazas a la seguridad nacional, el presidente desconoció al grupo de las FarC como un interlocutor político válido y embarcó al país en la guerra contra el terrorismo (iniciada por el presidente de los Estados Unidos George W. Bush desde los ataques del 11 de Septiembre de 2001) de manera que, bajo un principio de responsabilidad común y compartida, encontrará en el sistema internacional el apoyo en la lucha contra la mayor amenaza terrorista interna y a partir de la instrumentalización de la política exterior, se robusteciera la política de Seguridad Democrática .

Uribe Vélez, consideró como única salida al Conflicto Armado Interno, la victoria militar sobre los grupos guerrilleros e intensificó la guerra fortaleciendo de manera operativa y armamentística a las Fuerzas Militares Colombianas y buscando debilitar a los grupos al margen de la ley que controlaban zonas del territorio nacional; sin embargo, el vacío institucional persistente permitió la consolidación de grupos como las Autodefensas Unidas de Colombia, no sólo tratándose de ese vacío sino del apoyo de las Fuerzas Militares a dichos grupos autodefensas (AUC), sintiéndose legitimadas con un apoyo local de las poblaciones rurales, además de la emergencia de otros actores como las Bandas Criminales (Bacrim) que potenciaron el CAI.

En el primer periodo de gobierno de Juan Manuel Santos (2010-2014) el objetivo central era mantener la política de Seguridad Democrática como el legado de su antecesor; pero justo después de asumir el poder, los medios especularon sobre negociaciones que el mandatario estaría llevando en secreto con el grupo de las Farc. "[...] Santos les recordaba a los colombianos que sólo él tenía "la llave que podría abrirle la puerta a la paz". Sólo la usaría si las Farc demostraban intenciones sinceras de negociar y formar parte de la política nacional como una fuerza legítima y no como una organización ilegal armada." (Borda y Gómez 2017: 216).

Al mismo tiempo, el Presidente comenzó a adaptar su política exterior para recuperar la confianza de América Latina sin comprometer las relaciones con sus aliados históricos. La construcción de un nuevo eje 
de acción exterior del país orientada a la Respice Omnia, permitió la diversificación de la agenda política y de cooperación con Washington, y simultáneamente aliviar las tensiones que había dejado Álvaro Uribe Vélez entre Colombia y sus vecinos.

En ese sentido, las giras de Santos por España, Alemania y Francia para el ingreso de Colombia a la Organización para la Cooperación y el Desarrollo Económico (OCDE), llevaron un mensaje de seguridad, inversión y desarrollo a través de la paz. Las visitas de Estado a Brasil, Chile, Argentina, Perú, panamá, Costa Rica, México y otros países de Centro América sirvieron también como plataforma para expresar la voluntad política de dar primacía a las relaciones con América Latina desde las oportunidades compartidas, para insertarse de forma económica y comercial en la región. Colombia activó su participación en encuentros multilaterales y promovió, por medio de la diplomacia pública, el cambio en la percepción de Colombia, de un "país problema" a un país de oportunidades y soluciones resaltando su posición privilegiada como país bisagra entre América del Sur y Estados Unidos.

Santos, cambia toda la orientación de su política al reconocimiento de un Conflicto Armado Interno, dejando de lado la lucha contra una supuesta amenaza terrorista y en noviembre de 2012 anunció al país las claras intenciones de iniciar las mesas de negociación de un proceso de paz con el grupo guerrillero. Este hecho generó consecuencias importantes como el inicio de las diferencias entre Santos y Uribe, quien se configuró como el más grande opositor a su gobierno.
En los primeros años de gobierno, Santos a través de la diplomacia presidencial, buscó generar un tipo de spill over del Conflicto Armado Interno, pronunciando discursos contundentes en escenarios internacionales con la promoción de las políticas de "seguridad democrática" como continuidad del legado de su antecesor. En contraste, para el $72^{\circ}$ Periodo de Sesiones Ordinarias de la Asamblea General de Naciones Unidas, Juan Manuel Santos logró que el mundo se interesara en los problemas internos de Colombia, a través de la Diplomacia para la Paz, bajo el principio de responsabilidad común y compartida, y reconociendo la efectividad de la Organización de Naciones Unidas en el cumplimiento de la misión para la cual fue creada: trascender hacía la proscripción definitiva del flagelo de la guerra.

Una vez inició el proceso de paz, Colombia necesitó apoyo internacional para dar legitimidad e impulso a la salida negociada del conflicto. A este proceso se unieron Cuba, Chile, Noruega y Venezuela como garantes para dar confianza a las partes en momentos de crisis. Si bien, la participación de actores internacionales era crucial para el éxito de la negociación, Santos dejó implícito que la participación estaría limitada y la internacionalización del proceso de paz sería contenida, a diferencia de los procesos y acuerdos de cooperación realizados por presidentes anteriores que permitían el intervencionismo de países como Estados Unidos en los procesos internos de Colombia. El Gobierno Colombiano

[...] convirtió a la Asamblea General de la ONU en un espacio para compartir sus logros y dificultades, para solicitar a la comunidad internacional que respetara el proceso de 
paz, para abogar por el derecho de Colombia a alcanzar la paz (El espectador 2013) y para solicitar cooperación para la financiación del posconflicto. (Borda y Gómez 2017: 225).

Para la negociación, el hecho de haber logrado la activación del mecanismo de monitoreo y verificación con acompañamiento internacional que garantizara el pleno cumplimiento de compromisos adquiridos, rompió con la ausencia de las organizaciones internacionales en el proceso de paz (Borda, 2013: 3), y el rol de la Organización tomó fuerza en la promoción de la cooperación internacional para la solución de problemas multidimensionales.

Santos promovió en la Asamblea General "[...] la idea de que el mundo debía respetar la soberanía jurídica del país para definir los mecanismos de investigación y juzgamiento de más de cincuenta años en conflicto" (Valero, 2013) activando todas las posturas de los Estados miembros a favor de la legalidalidad y de la cooperación que Colombia requería para alcanzar la paz con éxito.

\section{Nadie es profeta en su tierra}

Santos supo aprovechar el compromiso internacional de actuar de conformidad con el discurso sólido de la paz y la seguridad de Colombia y la región. Las plataformas ya construidas reflejadas en nuevas alianzas bajo su liderazgo se convirtieron en el "castillo de naipes" de una popularidad cuestionada. Sin embargo, el apoyo internacional en favor de la paz aunado a la consistencia de los discursos de los mandatarios aliados en favor del acuerdo de paz con las Farc, le merecerían el premio político más importante de la historia del CAI colombiano: El Nobel de Paz.
La búsqueda del derrumbe de la popularidad del "Santo Legado" como misión de la oposición, inicia como un aspecto de la longevidad en el liderazgo y de los caudillismos en Colombia. No obstante, el pilar discutido en su momento sobre la negociación de La Habana fue: si con el apoyo ganado internacionalmente, mediante un liderazgo magistral ante la ONU y sus vecinos garantes, sería esto suficiente para convencer al pueblo colombiano durante el proceso de refrendación popular. Resulta apenas lógico que el extenso lapso de vida política del expresidente Álvaro Uribe fuera un obstáculo para la credibilidad del proceso de negociación al momento de refrendación.

"El Plebiscito por la Paz" fue el punto de inflexión en la popularidad del legado de Juan Manuel Santos. A partir de allí, diferentes encuestas sobre la gestión de su gobierno y la favorabilidad de su imagen ante la implementación de los acuerdos, arrojaron resultados que no ubicaron la buena imagen de Santos por encima del $40 \%$, teniendo en cuenta las poco satisfactorias fichas técnicas de las encuestas nacionales.

A diferencia de la baja opinión favorable con la que terminó Juan Manuel Santos al interior del país, en América Latina, la encuesta sobre las percepciones de líderes de opinión hacía presidentes, realizada por Ipsos Public Afairs en marzo de 2018, demostró que el Presidente Santos contaba con el mayor porcentaje obtenido entre los mandatarios de la región, distanciándose considerablemente de los resultados obtenidos a nivel nacional.

En esta encuesta aplicada a líderes de opinión latinoamericanos, se respondió a la pregunta: ¿qué tan favorable o desfavorable es su opinión de los siguientes gobernantes? 
y a partir de los resultados, se construyó un ranking de favorabilidad de los jefes de Estado de la región encabezado por Juan Manuel Santos con el 79\%, seguido de Tabaré Vázquez de Uruguay (78\%), Mauricio Macri de Argentina (72\%) y Lenín Moreno de Ecuador (71\%).

Juan Manuel Santos, en sus 8 años de gobierno, asumió el reto de dejarle al País un legado: "El Legado de la Paz". El presidente soñó en su momento con convertir a Colombia en un actor protagonista en América Latina y el Caribe, pero volcó todos sus esfuerzos en alcanzar la paz de un Estado sobreviviente de un conflicto de más de medio siglo. Con el desarrollo de la diplomacia presidencial como instrumento, se erigió como aquel presidente que había logrado un proceso de negociación sin precedentes. A pesar de ello, en Colombia sus planes y estrategias no fueron suficientes para derrotar la propaganda del "presidente que nos devolvió el país".

\section{Referencias bibliográficas}

Ipsos Public Affairs (2018) Encuesta a Lideres de Opinión de Latinoamérica: Imagen de Presidentes. (En línea) Recuperado de: https://www.ipsos. com/es-pe/encuesta-lideres-de-opinion-de-latinoamerica-imagen-de-presidentes

Pastrana, E. y Vera, D. (2012). Estrategias de la política exterior de Colombia en su calidad de potencia secundaria de Suramérica. En E. Pastrana, S. Jost y D. Flemes (Eds.), Colombia y Brasil: ¿socios estratégicos en la construcción de Suramérica? (pp. 187-235). Bogotá: Konrad Adenauer
Stiftung, GIGA y Universidad Javeriana.

Borda, S. y Gomez, S. (2017) La internacionalización del Proceso de Paz en la Habana: del aislamiento a la convención. EN Ticnker, A., y Bitar, S. (2017) Nuevos Enfoques para el Estudio de las Relaciones Internacionales de Colombia. Bogotá: Ediciones UniAndes. pp. 213-238.

Valero, D. (7 de septiembre de 2013). Colombia Busca Blindar en la ONU el Proceso con las FARC. El Tiempo. Recuperado de http:// 